TRANSACTIONS OF THE

AMERICAN MATHEMATICAL SOCIETY

Volume 360, Number 11, November 2008, Pages 5999-6015

S 0002-9947(08)04701-6

Article electronically published on June 26, 2008

\title{
THE STEINBERG SYMBOL AND SPECIAL VALUES OF $L$-FUNCTIONS
}

\author{
CECILIA BUSUIOC
}

\begin{abstract}
The main results of this article concern the definition of a compactly supported cohomology class for the congruence group $\Gamma_{0}\left(p^{n}\right)$ with values in the second Milnor $K$-group (modulo 2-torsion) of the ring of $p$-integers of the cyclotomic extension $\mathbb{Q}\left(\mu_{p^{n}}\right)$. We endow this cohomology group with a natural action of the standard Hecke operators and discuss the existence of special Hecke eigenclasses in its parabolic cohomology. Moreover, for $n=1$, assuming the non-degeneracy of a certain pairing on $p$-units induced by the Steinberg symbol when $(p, k)$ is an irregular pair, i.e. $p \mid \frac{B_{k}}{k}$, we show that the values of the above pairing are congruent $\bmod p$ to the $L$-values of a weight $k$, level 1 cusp form which satisfies Eisenstein-type congruences mod $p$, a result that was predicted by a conjecture of R. Sharifi.
\end{abstract}

\section{INTRODUCTION}

Let $p^{n}>1$ be a power of a positive prime $p, R_{n}:=\mathbb{Z}\left[\mu_{p^{n}}, \frac{1}{p}\right]$, and $G_{n}:=$ $\operatorname{Gal}\left(\mathbb{Q}\left(\mu_{p^{n}}\right) / \mathbb{Q}\right)$. Then $G_{n}$ acts naturally on Milnor's $K$-group, $K_{2}^{M}\left(R_{n}\right)$. We let the congruence group $\Gamma_{0}:=\Gamma_{0}\left(p^{n}\right)$ act on $K_{2}^{M}\left(R_{n}\right)$ via the homomorphism $\Gamma_{0} \rightarrow G_{n}$ sending $\gamma \in \Gamma_{0}$ to $\sigma_{a}$ where $a$ is the upper left hand entry of $\gamma$ and $\sigma_{a} \in G_{n}$ is given by $\sigma_{a}(\zeta)=\zeta^{a}$ for every $\zeta \in \mu_{p^{n}}$. In this paper, we will define a modular symbol

$$
\phi_{n} \in H_{c}^{1}\left(\Gamma_{0}, \widetilde{K}_{2}\left(R_{n}\right)\right),
$$

where $\widetilde{K}_{2}\left(R_{n}\right)=K_{2}^{M}\left(R_{n}\right)\left(\bmod 2\right.$-torsion). We note that the action of $G_{n}$ on $R_{n}$ induces a natural action of $G_{n}$ on the above cohomology group. Moreover, we will endow this cohomology group with a natural action of the Hecke operators $T_{\ell}$, $\ell \neq p$, and will prove the following theorem.

Theorem 1.1. Let $\varphi_{n} \in H_{\text {par }}^{1}\left(\Gamma_{0}, \widetilde{K}_{2}\left(R_{n}\right)\right)$ be the image of $\phi_{n}$ under the canonical map. Then

(1) $\varphi_{n} \mid T_{2}=\left(\sigma_{2}+2\right) \varphi_{n}$ if $p \neq 2$; and

(2) $\varphi_{n} \mid T_{3}=\left(\sigma_{3}+3\right) \varphi_{n}$ if $p \neq 3$.

Now set $n=1$, let $k \geq 2$ be an even integer and suppose $p>3$. Let $R=R_{1}$, $G=G_{1}$. Let $E=R^{\times} / R^{\times p}$ be the group of units modulo $p^{\text {th }}$ powers of units in $R$. Then we may decompose $E$ as a direct sum

$$
E=\bigoplus_{i=0}^{p-2} E^{(1-i)},
$$

Received by the editors October 27, 2006.

2000 Mathematics Subject Classification. Primary 11F67.

(C)2008 American Mathematical Society 5999 Reverts to public domain 28 years from publication 


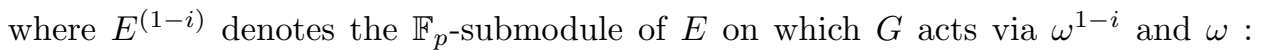
$G \longrightarrow \mathbb{F}_{p}^{\times}$is the canonical isomorphism. It is well known that $E^{(1)}=\mu_{p}$, and $E^{(1-i)}=0$ for even $i$ satisfying $0<i \leq p-3$. For $i$ odd, let $\eta_{i} \in E^{(1-i)}$ be the image of the cyclotomic $p$-unit $1-\zeta_{p}$ under the canonical projection $R^{\times} \longrightarrow E^{(1-i)}$. For $i=1,3, \ldots, p-2$, let $\xi_{i}=\left\{\eta_{k-i}, \eta_{i}\right\} \in\left(K_{2}^{M}(R) / p K_{2}^{M}(R)\right)^{(2-k)}$. We remark that Vandiver's conjecture implies that the cyclotomic $p$-units generate $E$, therefore also that the symbols $\xi_{i}(i=1, \ldots, p-2)$ generate $\left(K_{2}^{M}(R) / p K_{2}^{M}(R)\right)^{(2-k)}$. In what follows, we will often assume the following hypothesis:

Hypothesis $\left(H_{k}\right)$ : There exists a non-zero $G$-equivariant map

$$
\rho: K_{2}^{M}(R) / p K_{2}^{M}(R) \rightarrow \mathbb{F}_{p}\left(\omega^{2-k}\right)
$$

and an odd integer $i \neq p$ with $1<i<k-1$ such that the pairing induced by the composition of $\rho$ with the restriction of the Steinberg Symbol on the $p$-units

$$
E^{(1+i-k)} \times E^{(1-i)} \rightarrow \mathbb{F}_{p}\left(\omega^{2-k}\right)
$$

is non-degenerate.

We have the following theorem.

Theorem 1.2. Let $p>3$ be a prime and $k$ an even integer, $2 \leq k<2 p$, for which hypothesis $H_{k}$ holds. Then there exists a non-zero parabolic cohomology class $\psi \in H_{\text {par }}^{1}\left(S L_{2}(\mathbb{Z}), S_{y m m}^{k-2}\left(\mathbb{F}_{p}^{2}\right)\right)$ such that

(1) $\psi \mid T_{q}=\left(1+q^{k-1}\right) \psi$ for $q=2,3$.

(2) For $3 \leq i \leq k-3, i \neq p$, we have $L(\psi, i)=\rho\left(\xi_{i}\right)$.

The above results were motivated by the joint work of W. McCallum and R. Sharifi [MS03, MS] and by a well-publicized conjecture of Sharifi (see [Sh1-04]). Indeed, McCallum and Sharifi MS03] predict that, assuming Vandiver's Conjecture, Hypothesis $H_{k}$ holds whenever $p$ is irregular with $p \mid \frac{B_{k}}{k}$. In this case, Sharifi's conjecture Sh1-04] predicts the truth of Theorem 1.2 with the Hecke condition (1) strengthened to include all primes $q \neq p$ (not just $q=2,3$ ). In future work of the author with G. Stevens, we will generalize Theorem 1.2 by constructing a universal Eisenstein cohomology class $\Psi$ that specializes to the class $\psi$ of Theorem 1.2. The general statement (1) about Hecke eigenvalues will follow from the properties of $\Psi$. Details will appear later.

Now, for a set $S$ of positive primes $q \neq p$, let

$$
H_{k, e i s, S}^{+} \subseteq H_{\text {par }}^{1}\left(S L_{2}(\mathbb{Z}), \operatorname{Symm}^{k-2}\left(\mathbb{F}_{p}\right)\right)
$$

denote the subspace of all vectors that are fixed by complex conjugation and on which the operators $T_{q}$ for $q \in S$ act with eigenvalue $1+q^{k-1}$. It follows from Theorem 1.2 that $H_{k, e i s,\{2,3\}}^{+}$is positive dimensional whenever hypothesis $H_{k}$ holds. The following theorem is a consequence of Theorem 1.2.

Theorem 1.3. Let $(p, k)$ be an irregular pair with $k<p$ such that hypothesis $H_{k}$ holds and assume $H_{k, e i s,\{2,3\}}^{+}$is one-dimensional. Then Sharifi's conjecture is true for the pair $(p, k)$.

Finally, we remark that Romyar Sharifi has recently and independently also proved Theorem 1.1.

I would like to mention that the present work would not have been possible without the insightful advice and wealth of ideas from my thesis advisor, Glenn 
Stevens. I am also extremely grateful for his immense help with the expository part of the paper and Lemma 7.5. I would also like to thank Romyar Sharifi for his generosity in sharing copies of transparencies from talks he has given on his conjectures and also for a number of helpful conversations.

\section{Modular Symbols}

Let $\Delta=\operatorname{Div}\left(\mathbb{P}^{1}(\mathbb{Q})\right)$ and $\Delta_{0} \subseteq \Delta$ be the subgroup of divisors of degree 0 . The group $G L_{2}(\mathbb{Q})$ acts by fractional linear transformations on $\Delta$ and $\Delta_{0}$. Let $\Sigma_{0}\left(p^{n}\right)=\left\{\left(\begin{array}{ll}a & b \\ c & d\end{array}\right) \in M_{2}^{+}(\mathbb{Z})\left|(a, p)=1, p^{n}\right| c\right\}$ and $\Sigma_{1}\left(p^{n}\right)=\left\{\left(\begin{array}{ll}a & b \\ c & d\end{array}\right) \in M_{2}^{+}(\mathbb{Z}) \mid a \equiv\right.$ $\left.1\left(\bmod p^{n}\right), p^{n} \mid c\right\}$.

For any right $\Sigma_{0}\left(p^{n}\right)$-module $M$ we define a right action of $\Sigma_{0}\left(p^{n}\right)$ on $\operatorname{Hom}_{\mathbb{Z}}\left(\Delta_{0}, M\right)$ by

$$
(\phi \mid \sigma)(D)=\phi(\sigma D) \mid \sigma
$$

for all $\sigma \in \Sigma_{0}\left(p^{n}\right), D \in \Delta_{0}$. The group of $M$-valued modular symbols over $\Gamma_{0}\left(p^{n}\right)$ is defined to be the group

$$
\operatorname{Symb}_{\Gamma_{0}\left(p^{n}\right)}(M):=\operatorname{Hom}_{\mathbb{Z}}\left(\Delta_{0}, M\right)^{\Gamma_{0}\left(p^{n}\right)} .
$$

For each positive integer $m$ we define the Hecke operator

$$
\begin{aligned}
T_{m}: \operatorname{Symb}_{\Gamma_{0}\left(p^{n}\right)}(M) & \longrightarrow \operatorname{Symb}_{\Gamma_{0}\left(p^{n}\right)}(M) \\
\phi & \longmapsto \phi\left|T_{m}:=\sum_{i} \phi\right| \delta_{i},
\end{aligned}
$$

where the sum is over a complete set of representatives $\left\{\delta_{i}\right\}_{i}$ for the left $\Gamma_{0}\left(p^{n}\right)$ cosets in the double coset $\Gamma_{0}\left(p^{n}\right)\left(\begin{array}{ll}1 & 0 \\ 0 & m\end{array}\right) \Gamma_{0}\left(p^{n}\right)$.

For a cusp $r \in \mathbb{P}^{1}(\mathbb{Q})$, let $\Gamma_{r}$ be the stabilizer subgroup in $\Gamma_{0}\left(p^{n}\right)$ of $r$. Then for any $m \in M^{\Gamma_{r}}$ we define $\widetilde{\phi}_{r, m}: \mathbb{P}^{1}(\mathbb{Q}) \rightarrow M$ by defining

$$
\widetilde{\phi}_{r, m}(s)= \begin{cases}m \mid \gamma^{-1} & \text { if } s=\gamma r, \text { with } \gamma \in \Gamma_{0}\left(p^{n}\right), \\ 0 & \text { otherwise. }\end{cases}
$$

We extend $\widetilde{\phi}_{r, m}$ by linearity to an additive function

$$
\widetilde{\phi}_{r, m}: \Delta \longrightarrow M
$$

and note that the restriction of $\widetilde{\phi}_{r, m}$ to $\Delta_{0}$ is an $M$-valued modular symbol over $\Gamma_{0}\left(p^{n}\right)$, which we denote $\phi_{r, m}$.

Definition 2.1. A modular symbol of the form $\phi_{r, m}$ will be called a boundary symbol supported on the $r$-cusps, i.e. the cusps that are $\Gamma_{0}\left(p^{n}\right)$-equivalent to $r$. We define the group of $M$-valued boundary symbols over $\Gamma_{0}\left(p^{n}\right)$ to be the subgroup

$$
\operatorname{Bound}_{\Gamma_{0}\left(p^{n}\right)}(M) \subseteq \operatorname{Symb}_{\Gamma_{0}\left(p^{n}\right)}(M)
$$

generated by the set of all $\phi_{r, m}$, where $r, m$ run over all pairs with $r \in \mathbb{P}^{1}(\mathbb{Q})$ and $m \in M^{\Gamma_{r}}$.

There is also a canonical map $\operatorname{Symb}_{\Gamma_{0}\left(p^{n}\right)}(M) \longrightarrow H^{1}\left(\Gamma_{0}\left(p^{n}\right), M\right)$. If $\phi \in$ $\operatorname{Symb}_{\Gamma_{0}\left(p^{n}\right)}(M)$ and $r \in \mathbb{P}^{1}(\mathbb{Q})$, then the map $\Gamma_{0}\left(p^{n}\right) \longrightarrow M$ defined by $\gamma \longmapsto$ $\phi((\gamma r)-(r))$ is a 1-cocycle, whose cohomology class is independent of the choice of $r$. We let $\pi_{\phi}$ be that cohomology class. From the definition it is clear that for any $r \in \mathbb{P}^{1}(\mathbb{Q})$, the restriction of $\pi_{\phi}$ to $\Gamma_{r}$ is trivial. Thus, we have

$$
\pi_{\phi} \in H^{1}\left(\Gamma_{0}\left(p^{n}\right), M\right) \text {. }
$$


We have the following theorem of Ash and Stevens AS86.

Theorem 2.2. If multiplication by 6 is invertible on $M$, then there is a canonical isomorphism $H_{c}^{1}\left(\Gamma_{0}\left(p^{n}\right), M\right) \cong \operatorname{Symb}_{\Gamma_{0}\left(p^{n}\right)}(M)$. Moreover, there is a canonical commutative diagram

$$
\begin{aligned}
& 0 \longrightarrow \operatorname{Bound}_{\Gamma_{0}\left(p^{n}\right)}(M) \longrightarrow \operatorname{Symb}_{\Gamma_{0}\left(p^{n}\right)}(M) \stackrel{\pi_{\phi}}{\longrightarrow} H_{p a r}^{1}\left(\Gamma_{0}\left(p^{n}\right), M\right) \longrightarrow 0 \\
& 0 \longrightarrow H_{\partial}^{1}\left(\Gamma_{0}\left(p^{n}\right), M\right) \longrightarrow H_{c}^{1}\left(\Gamma_{0}\left(p^{n}\right), M\right) \longrightarrow H_{p a r}^{1}\left(\Gamma_{0}\left(p^{n}\right), M\right) \longrightarrow 0
\end{aligned}
$$

in which the rows are exact, the vertical arrows are isomorphisms, and all maps commute with the natural action of the Hecke operators $T_{m}(m \in \mathbb{N})$. Here, $H_{\partial}^{1}$ is the "boundary cohomology", which is defined by the exactness of the second row.

\section{MANin SYMBOLS}

In the special case where the subgroup $\Gamma_{1}\left(p^{n}\right) \subseteq \Gamma_{0}\left(p^{n}\right)$ acts trivially on $M$ we can give a simple description of $\operatorname{Symb}_{\Gamma_{0}\left(p^{n}\right)}(M)$ in terms of "Manin symbols". We recall that description in this section.

Let $A$ be a commutative ring. A group homomorphism

$$
\chi: \Gamma_{0}\left(p^{n}\right) \longrightarrow A^{\times}
$$

will be called a nebentype character if $\chi$ is trivial on $\Gamma_{1}\left(p^{n}\right)$. Any nebentype character $\chi$ extends uniquely to a multiplicative map

$$
\chi: \Sigma_{0}\left(p^{n}\right) \longrightarrow A^{\times}
$$

that is trivial on $\Sigma_{1}\left(p^{n}\right)$.

Definition 3.1. Let $A$ be a ring and $M$ be an $A$-module endowed with a right action of $\Sigma_{0}\left(p^{n}\right)$. We say that $\Sigma_{0}\left(p^{n}\right)$ acts via the nebentype character $\chi$ if for all $\gamma \in \Sigma_{0}\left(p^{n}\right)$ and all $m \in M$ we have $m \mid \gamma=\chi(\gamma) \cdot m$.

The group $S L_{2}(\mathbb{Z})$ acts by right matrix multiplication on the additive group of row vectors $\left(\mathbb{Z} / p^{n} \mathbb{Z}\right)^{2}$. The orbit of $(0,1)$ is the set

$$
X_{n}:=\left(\left(\mathbb{Z} / p^{n} \mathbb{Z}\right)^{2}\right)^{\prime}=\left\{(x, y) \in\left(\mathbb{Z} / p^{n} \mathbb{Z}\right)^{2} \mid(x, y)=(1)\right\} .
$$

The stabilizer of $(0,1)$ is the subgroup $\Gamma_{1}\left(p^{n}\right)$, which is a normal subgroup of $\Gamma_{0}\left(p^{n}\right)$. Thus $\Gamma_{0}\left(p^{n}\right)$ also acts on $X_{n}$ on the left. In fact, if $\gamma=\left(\begin{array}{ll}a & b \\ c & d\end{array}\right)$, then the left action of $\gamma$ on $X_{n}$ is given by scalar multiplication by $d: \gamma \mathbf{x} \mapsto d \cdot \mathbf{x}$. For the rest of this section $M$ will be an $A$-module on which $\Sigma_{0}\left(p^{n}\right)$ acts via the nebentype character $\chi: \Sigma_{0}\left(p^{n}\right) \longrightarrow A^{\times}$.

Definition 3.2. A function

$$
e: X_{n} \longrightarrow M
$$

is called an $M$-valued Manin symbol over $\Gamma_{0}\left(p^{n}\right)$ if $e$ satisfies the following "Manin relations" for all $\mathbf{x}=(x, y) \in X_{n}$ and $\lambda \in\left(\mathbb{Z} / p^{n} \mathbb{Z}\right)^{\times}$:

(1) $e(\lambda \mathbf{x})=\chi(\lambda) \cdot e(\mathbf{x})$;

(2) $e(x, y)+e(y,-x)=0$; and

(3) $e(x, y)+e(y,-x-y)+e(-x-y, x)=0$.

We denote by

$$
\operatorname{Manin}_{\Gamma_{0}\left(p^{n}\right)}(M)
$$

the group of all $M$-valued Manin symbols over $\Gamma_{0}\left(p^{n}\right)$. 
Remark 3.3. Two applications of the second Manin condition show that if $e$ is a Manin symbol, then $e(\mathbf{x})=e(-\mathbf{x})$ for every $\mathbf{x} \in X_{n}$. So if $e \neq 0$, then the first condition implies $\chi$ must be even, i.e. $\chi(-1)=1$.

Now fix a section $X_{n} \longrightarrow S L_{2}(\mathbb{Z}), \mathbf{x} \mapsto \gamma_{\mathbf{x}}$, so that

$$
(0,1) \gamma_{\mathbf{x}}=\mathbf{x}
$$

for every $\mathbf{x} \in X_{n}$. Also let $D_{\mathbf{x}} \in \Delta_{0}$ be given by

$$
D_{\mathbf{x}}:=\gamma_{\mathbf{x}} \cdot((\infty)-(0)) .
$$

If $M$ is a right $\Gamma_{0}\left(p^{n}\right)$-module and $\phi \in \operatorname{Symb}_{\Gamma_{0}\left(p^{n}\right)}(M)$, then we define $\mathbf{e}_{\phi}: X_{n} \longrightarrow$ $M$ by

$$
\mathbf{e}_{\phi}(\mathbf{x}):=\phi\left(D_{\mathbf{x}}\right)
$$

and note that this is well defined independent of our choices of the $\gamma_{\mathbf{x}}$. We have the following reformulation of a theorem of Manin Ma72.

Theorem 3.4. The map $\phi \longmapsto \mathbf{e}_{\phi}$ induces an isomorphism

$$
\mathbf{e}: \operatorname{Symb}_{\Gamma_{0}\left(p^{n}\right)}(M) \longrightarrow \operatorname{Manin}_{\Gamma_{0}\left(p^{n}\right)}(M)
$$

for every right $\Gamma_{0}\left(p^{n}\right)$-module $M$.

We use this isomorphism to transfer the action of the Hecke operators $T_{m}$ on $\operatorname{Symb}_{\Gamma_{0}\left(p^{n}\right)}(M)$ to an action on $\operatorname{Manin}_{\Gamma_{0}\left(p^{n}\right)}(M)$. The following theorem of Merel Mer94 gives an "explicit" description of this action.

Theorem 3.5. Let $m$ be a positive integer and let

$$
H_{m}:=\left\{\begin{array}{ll}
\left(\begin{array}{ll}
a & b \\
c & d
\end{array}\right) & \begin{array}{l}
a, b, c, d \in \mathbb{Z} \\
a>b \geq 0, d>c \geq 0 \\
a d-b c=m
\end{array}
\end{array}\right\} .
$$

Then for every $e \in \operatorname{Manin}_{\Gamma_{0}\left(p^{n}\right)}(M)$ we have

$$
\left(e \mid T_{m}\right)(\mathbf{x})=\sum_{\delta \in H_{m}} e(\mathbf{x} \delta)
$$

For future reference we record the following corollary.

Corollary 3.6. For arbitrary $e \in \operatorname{Manin}_{\Gamma_{0}\left(p^{n}\right)}(M)$ we have

$$
\begin{aligned}
\left(e \mid T_{2}\right)(\mathbf{x})= & e(x, 2 y)+e(2 x, y)+e(x+y, 2 y)+e(2 x, x+y), \\
\left(e \mid T_{3}\right)(\mathbf{x})= & e(x, 3 y)+e(3 x, y)+e(x+y, 3 y)+e(3 x, x+y) \\
& +e(x-y, 3 y)+e(3 x, x-y) .
\end{aligned}
$$

Proof. We easily verify that

$$
\begin{aligned}
& H_{2}=\left\{\left(\begin{array}{ll}
1 & 0 \\
0 & 2
\end{array}\right),\left(\begin{array}{ll}
2 & 0 \\
0 & 1
\end{array}\right),\left(\begin{array}{ll}
1 & 0 \\
1 & 2
\end{array}\right),\left(\begin{array}{ll}
2 & 1 \\
0 & 1
\end{array}\right)\right\} \text { and } \\
& H_{3}=\left\{\left(\begin{array}{ll}
1 & 0 \\
0 & 3
\end{array}\right),\left(\begin{array}{ll}
3 & 0 \\
0 & 1
\end{array}\right),\left(\begin{array}{ll}
1 & 0 \\
1 & 3
\end{array}\right),\left(\begin{array}{ll}
1 & 0 \\
2 & 3
\end{array}\right),\left(\begin{array}{ll}
3 & 1 \\
0 & 1
\end{array}\right),\left(\begin{array}{ll}
3 & 2 \\
0 & 1
\end{array}\right),\left(\begin{array}{ll}
2 & 1 \\
1 & 2
\end{array}\right)\right\} .
\end{aligned}
$$

The description of $T_{2}$ is then an immediate consequence of Merel's theorem. On the other hand, Merel's theorem implies

$$
\begin{aligned}
\left(e \mid T_{3}\right)(\mathbf{x})= & e(x, 3 y)+e(3 x, y)+e(x+y, 3 y)+e(3 x, x+y) \\
& +e(x+2 y, 3 y)+e(3 x, 2 x+y)+e(2 x+y, 2 y+x) .
\end{aligned}
$$


Now consider the following matrix:

$$
\left(\begin{array}{ccc}
e(x+2 y, 3 y) & e(x-y, x+2 y) & 0 \\
e(3 x, 2 x+y) & 0 & e(2 x+y, x-y) \\
e(2 x+y, x+2 y) & e(x+2 y, x-y) & e(x-y, 2 x+y)
\end{array}\right) .
$$

From the Manin relations, we see that the sum of the three rows are $e(x-y, 3 y)$, $e(3 x, x-y)$, and 0 , respectively. Thus the sum of all the entries of the matrix is $e(x-y, 3 y)+e(3 x, x-y)$. On the other hand, the second and third columns sum to 0 . Hence the sum of all the entries of the matrix is the sum of the first column. We therefore have

$$
e(x+2 y, 3 y)+e(3 x, 2 x+y)+e(2 x+y, x+2 y)=e(x-y, 3 y)+e(3 x, x-y) .
$$

Substituting this into the above expression for $e \mid T_{3}$ gives us

$$
\begin{aligned}
\left(e \mid T_{3}\right)(\mathbf{x})= & e(x, 3 y)+e(3 x, y)+e(x+y, 3 y)+e(3 x, x+y) \\
& +e(x-y, 3 y)+e(3 x, x-y)
\end{aligned}
$$

proving our claim for $e \mid T_{3}$.

We conclude this section with a discussion of boundary symbols supported on the $\infty$-cusps (see Definition 2.1).

Definition 3.7. We say that a Manin symbol $e \in \operatorname{Manin}_{\Gamma_{0}\left(p^{n}\right)}(M)$ is supported at $\infty$ if $e$ satisfies the following condition:

$$
e(\mathbf{x})=0 \quad \text { for all } \mathbf{x}=(x, y) \in X_{n} \text { with } x y \neq 0 .
$$

We denote by

$$
\operatorname{Manin}_{\Gamma_{0}\left(p^{n}\right)}^{\infty}(M) \subseteq \operatorname{Manin}_{\Gamma_{0}\left(p^{n}\right)}(M)
$$

the subgroup of all Manin symbols supported at $\infty$.

It is easy to describe the action of the Hecke operators on $\operatorname{Manin}_{\Gamma_{0}\left(p^{n}\right)}^{\infty}(M)$. The result is the following.

Proposition 3.8. The subgroup $\operatorname{Manin}_{\Gamma_{0}\left(p^{n}\right)}^{\infty}(M)$ is an eigen-submodule for the action of the Hecke operators $T_{m}$ on $\operatorname{Manin}_{\Gamma_{0}\left(p^{n}\right)}(M)$. Moreover, we have:

- if $\ell \neq p$ is prime, then the eigenvalue of $T_{\ell}$ is $\ell+\chi(\ell)$;

- the eigenvalue of $T_{p}$ is $p$.

The proof is an easy computation from the definitions. We note that the formal Dirichlet series of Hecke acting on $\operatorname{Manin}_{\Gamma_{0}\left(p^{n}\right)}^{\infty}(M)$ is given by the formal identity

$$
\sum_{m=1}^{\infty} T_{m} m^{-s}=\left(1-p^{1-s}\right)^{-1} \prod_{\ell \neq p}\left(1-\ell^{1-s}\right)^{-1}\left(1-\chi(\ell) \ell^{-s}\right)^{-1}
$$

which we may write suggestively in the form

$$
\sum_{m=1}^{\infty} T_{m} m^{-s}=\zeta(s-1) \cdot L(s, \chi) .
$$

The right hand side is the $L$-function of an Eisenstein series $E_{\chi}$ defined over the ring $A$. 


\section{4. $K_{2}$ OF CYCLOTOMIC INTEGER RINGS}

First, we recall the definition of Milnor's $K_{2}$-group of a commutative ring $R$.

Definition 4.1. The second Milnor $K$-group of $R$ is defined as

$$
K_{2}^{M}(R):=\left(R^{\times} \otimes_{\mathbb{Z}} R^{\times}\right) / I_{2}
$$

where $I_{2}$ is the subgroup of $R^{\times} \otimes_{\mathbb{Z}} R^{\times}$generated by the set

$$
\left\{a_{1} \otimes a_{2} \in R^{\times} \otimes_{\mathbb{Z}} R^{\times} \mid a_{1}+a_{2} \in\{0,1\}\right\} .
$$

The Steinberg Symbol is defined as the canonical map

$$
\{,\}: R^{\times} \times R^{\times} \longrightarrow K_{2}^{M}(R) .
$$

We write the multiplication in $K_{2}^{M}(R)$ additively and note that for $a, b \in R^{\times}$we have

$$
\{a, b\}+\{b, a\}=0
$$

as an easy consequence of the relation $\{a b,-a b\}=0$. This skew-symmetry of the Steinberg symbol will be used throughout the paper.

Denote by $\mu_{n}$ the group of $p^{n}$-th roots of unity in $\mathbb{C}^{\times}$and fix $\zeta_{n} \in \mu_{n}$ a primitive $p^{n}$-th root of unity. Let $K_{n}:=\mathbb{Q}\left(\mu_{n}\right), R_{n}=\mathbb{Z}\left[\mu_{n}, \frac{1}{p}\right]$, and $G_{n}:=\operatorname{Gal}\left(K_{n} / \mathbb{Q}\right)$. We associate to each $a \in\left(\mathbb{Z} / p^{n} \mathbb{Z}\right)^{\times}$the element $\sigma_{a} \in G_{n}$ for which $\zeta_{n}^{\sigma_{a}}=\zeta^{a}$. As in the introduction, we let $\widetilde{K}_{2}\left(R_{n}\right)=K_{2}^{M}\left(R_{n}\right) /(2$-torsion $)$. Note that $\widetilde{K}_{2}\left(R_{n}\right)$ has a natural action of the Galois group $G_{n}$.

In the next section, we will need the following lemma.

Lemma 4.2. For any $x, y \in \mathbb{Z} / p^{n} \mathbb{Z}$ with $x \neq 0$ we have

$$
\left\{1-\zeta_{n}^{x}, \zeta_{n}^{y}\right\}=0 .
$$

Proof. If $x \in\left(\mathbb{Z} / p^{n} \mathbb{Z}\right)^{\times}$, then we may choose $a \in \mathbb{Z}$ such that $a x=1$ in $\left(\mathbb{Z} / p^{n} \mathbb{Z}\right)^{\times}$. But then $\left\{1-\zeta_{n}^{x}, \zeta_{n}^{y}\right\}=\left\{1-\zeta_{n}^{x}, \zeta_{n}^{a x y}\right\}=a y \cdot\left\{1-\zeta_{n}^{x}, \zeta_{n}^{x}\right\}=0$, proving the lemma in this special case. In the general case we may write $x \equiv p^{k} u\left(\bmod p^{n}\right)$ for $u, k \in \mathbb{Z}$ with $p \nmid u$ and $0 \leq k<n$. Then,

$$
1-\zeta_{n}^{x}=\prod_{\substack{\alpha \in\left(\mathbb{Z} / p^{n} \mathbb{Z}\right)^{\times} \\ \alpha \equiv 1\left(p^{n-k}\right)}} 1-\zeta_{n}^{u \alpha},
$$

and the relation will now follow from the special case.

$$
\text { 5. A } \widetilde{K}_{2}\left(R_{n}\right) \text {-VALUed MANin SYMBOL }
$$

Define the Artin nebentype character to be the character

$$
\begin{aligned}
\chi: \Sigma_{0}\left(p^{n}\right) & \longrightarrow \mathbb{Z}\left[G_{n}\right]^{\times} \\
\gamma & \longmapsto \sigma_{a},
\end{aligned}
$$

where, as always, $a$ is the upper left corner of $\gamma$. We let $\Sigma_{0}\left(p^{n}\right)$ act on $\widetilde{K}_{2}\left(R_{n}\right)$ via the Artin nebentype $\chi$.

Theorem 5.1. The function $e_{n}: X_{n} \longrightarrow \widetilde{K}_{2}\left(R_{n}\right)$ defined by

$$
e_{n}(x, y)= \begin{cases}\left\{1-\zeta_{n}^{x}, 1-\zeta_{n}^{y}\right\} & \text { if } x, y \neq 0 \\ 0 & \text { otherwise }\end{cases}
$$


is a Manin symbol: $e_{n} \in \operatorname{Manin}_{\Gamma_{0}\left(p^{n}\right)}\left(\widetilde{K}_{2}\left(R_{n}\right)\right)$. Moreover, for $q=2,3$,

$$
e_{n} \mid\left(T_{q}-(q+\chi(q))\right) \in \operatorname{Manin}_{\Gamma_{0}\left(p^{n}\right)}^{\infty}\left(\widetilde{K}_{2}\left(R_{n}\right)\right) .
$$

Proof. We begin by verifying that $e_{n}$ satisfies the three Manin conditions (see Definition 3.2). Let $\mathbf{x}=(x, y) \in X_{n}$ be fixed and note that the first two Manin conditions are trivially satisfied if $x y=0$.

For $\lambda \in\left(\mathbb{Z} / p^{n} \mathbb{Z}\right)^{\times}$we have

$$
\begin{aligned}
e_{n}(\lambda \mathbf{x}) & =\left\{1-\zeta_{n}^{\lambda x}, 1-\zeta_{n}^{\lambda y}\right\}=\left\{1-\zeta_{n}^{x}, 1-\zeta_{n}^{y}\right\}^{\sigma_{\lambda}} \\
& =\chi(\lambda) \cdot e_{n}(\mathbf{x}) .
\end{aligned}
$$

So the first Manin condition is satisfied.

We let $\sim$ denote the congruence modulo 2-torsion in $K_{2}^{M}\left(R_{n}\right)$ (recall that $\widetilde{K}_{2}\left(R_{n}\right)$ $=K_{2}^{M}\left(R_{n}\right) /(2$-torsion $\left.)\right)$. From the properties of the Steinberg symbol we have, for $x y \neq 0$,

$$
\begin{aligned}
e_{n}(x, y) & \sim\left\{\zeta_{n}^{x}-1,1-\zeta_{n}^{y}\right\}=-\left\{1-\zeta_{n}^{y}, \zeta_{n}^{x}-1\right\} \\
& =-\left\{1-\zeta_{n}^{y}, \zeta_{n}^{x}\right\} \cdot\left\{1-\zeta_{n}^{y}, 1-\zeta_{n}^{-x}\right\} \\
& =-\left\{1-\zeta_{n}^{y}, 1-\zeta_{n}^{-x}\right\} \\
& =-e_{n}(y,-x) .
\end{aligned}
$$

We used Lemma 4.2 to derive the second to last equality. This proves the second Manin condition.

To verify the third Manin condition,

$$
e_{n}(x, y)+e_{n}(y,-x-y)+e_{n}(-x-y, x)=0,
$$

we consider cases. If $x=0$, then $y \neq 0$, and in that case $e_{n}(y,-y)=-e_{n}(y, y)$ by the second Manin condition. But by the skew-symmetry of the Steinberg symbol we have $e_{n}(y, y) \sim 0$, so $e_{n}(y,-y) \sim 0$ and the third Manin condition is satisfied in this case. Similarly, it is satisfied if either $y=0$ or $x+y=0$. So we may assume $x, y$, and $x+y$ are all non-zero. In this case, we have the identity

$$
\frac{\zeta_{n}^{y}\left(1-\zeta_{n}^{x}\right)}{1-\zeta_{n}^{x+y}}+\frac{1-\zeta_{n}^{y}}{1-\zeta_{n}^{x+y}}=1
$$

From the Steinberg relations we then have

$$
\left\{\frac{\zeta_{n}^{y}\left(1-\zeta_{n}^{x}\right)}{1-\zeta_{n}^{x+y}}, \frac{1-\zeta_{n}^{y}}{1-\zeta_{n}^{x+y}}\right\}=0
$$

Bimultiplicativity of the Steinberg symbol, Lemma 4.2 , and the skew symmetry of $e_{n}$ imply

$$
e_{n}(x, y)-e_{n}(x+y, y)-e_{n}(x, x+y)=0 .
$$

Now apply the second Manin condition to the last two terms to obtain

$$
e_{n}(x, y)+e_{n}(y,-x-y)+e_{n}(-x-y, x)=0,
$$

and the third Manin condition is proved. This proves $e_{n}$ is a Manin symbol.

To compute the Hecke operators, we use Corollary 3.6 to Merel's Theorem 3.5

Lemma 5.2. Let $q=2$ or $q=3$. Then for all $\mathbf{x}=(x, y)$ with $x y \neq 0$ we have

$$
\left(e_{n} \mid T_{q}\right)(\mathbf{x})=(q+\chi(q)) \cdot e_{n}(\mathbf{x}) .
$$


Proof. For $q=2$ we have, from Corollary 3.6.

$$
\left(e_{n} \mid T_{2}\right)(x, y)=e_{n}(x, 2 y)+e_{n}(2 x, y)+e_{n}(x+y, 2 y)+e_{n}(2 x, x+y) .
$$

If $x+y=0$ this says $\left(e_{n} \mid T_{2}\right)(x,-x)=e_{n}(x,-2 x)+e_{n}(2 x,-x)$, which vanishes by skew symmetry and the second Manin condition. But also $e_{n}(x,-x)=0$, so we have

$$
\left(e_{n} \mid T_{2}\right)(x,-x)=(2+\chi(2)) \cdot e_{n}(x,-x)
$$

since both sides of this equation vanish.

If $x+y \neq 0$, then we use the following identity, which was discovered by W. McCallum and R. Sharifi MS03:

$$
\frac{\left(1-\zeta_{n}^{x+y}\right)\left(1-\zeta_{n}^{x}\right)}{1-\zeta_{n}^{2 x}}+\zeta_{n}^{x} \frac{\left(1-\zeta_{n}^{2 y}\right)\left(1-\zeta_{n}^{x}\right)}{\left(1-\zeta_{n}^{2 x}\right)\left(1-\zeta_{n}^{y}\right)}=1 .
$$

This implies

$$
\begin{aligned}
& e_{n}(x, 2 y)+e_{n}(2 x, y)+e_{n}(x+y, 2 y)-e_{n}(x+y, 2 x) \\
& \quad=e_{n}(x, y)+e_{n}(2 x, 2 y)+e_{n}(x+y, y)-e_{n}(x+y, x)+e_{n}(x, 2 x)+e_{n}(2 x, x) .
\end{aligned}
$$

According to the above, the left hand side of this equality is $\left(e_{n} \mid T_{2}\right)(x, y)$. So we have

$$
\begin{aligned}
\left(e_{n} \mid T_{2}\right)(x, y)= & 2 e_{n}(x, y)+e_{n}(2 x, 2 y) \\
& -\left(e_{n}(x, y)+e_{n}(y, x+y)+e_{n}(x+y, x)\right) \\
& +\left(e_{n}(x, 2 x)+e_{n}(2 x, x)\right) .
\end{aligned}
$$

But the last two lines of the right side of this equation vanish by the third and second Manin conditions, so we have

$$
\left(e_{n} \mid T_{2}\right)(\mathbf{x})=(2+\chi(2)) \cdot e_{n}(\mathbf{x})
$$

and the assertion for $T_{2}$ is proved.

For $q=3$ we again use Corollary 3.6 to obtain

$$
\begin{aligned}
\left(e_{n} \mid T_{3}\right)(x, y)= & e_{n}(x, 3 y)+e_{n}(3 x, y)+e_{n}(x+y, 3 y) \\
& +e_{n}(x-y, 3 y)+e_{n}(3 x, x+y)+e_{n}(3 x, x-y) .
\end{aligned}
$$

If $x+y=0$, then the right hand side simplifies to $e_{n}(x,-3 x)+e_{n}(3 x,-x)+$ $e_{n}(-x,-3 x)+e_{n}(3 x, x)$, which vanishes by the skew symmetry of $e_{n}$. A similar calculation shows that the right hand side vanishes when $x-y=0$. Thus in either case, we have

$$
\left(e_{n} \mid T_{3}\right)(x, \pm x)=(3+\chi(3)) \cdot e_{n}(x, \pm x)
$$

since both sides vanish.

So we may assume $x+y, x-y \neq 0$. In that case, we have the following identity (see [Sh2-04):

$$
\zeta_{n}^{y-x}\left(\zeta_{n}^{2 x}+\zeta_{n}^{x}+1\right)+\left(1-\zeta_{n}^{y-x}\right)\left(1-\zeta_{n}^{x+y}\right)=\frac{1-\zeta_{n}^{3 y}}{1-\zeta_{n}^{y}}
$$

which may be rewritten as

$$
\frac{\zeta_{n}^{y-x}\left(1-\zeta_{n}^{3 x}\right)\left(1-\zeta_{n}^{y}\right)}{\left(1-\zeta_{n}^{x}\right)\left(1-\zeta_{n}^{3 y}\right)}+\frac{\left(1-\zeta_{n}^{y-x}\right)\left(1-\zeta_{n}^{y}\right)\left(1-\zeta_{n}^{x+y}\right)}{1-\zeta_{n}^{3 y}}=1 .
$$


This implies

$$
\begin{gathered}
e_{n}(x, 3 y)+e_{n}(3 x, y)-e_{n}(3 y, x+y)-e_{n}(3 y, y-x)+e_{n}(3 x, x+y) \\
+e_{n}(3 x, y-x)=e_{n}(3 x, 3 y)-e_{n}(y, y-x)-e_{n}(y, x+y)+e_{n}(x, y-x) \\
+e_{n}(x, y)+e_{n}(x, x+y) .
\end{gathered}
$$

The left hand side of this equality is $\left(e_{n} \mid T_{3}\right)(x, y)$. So we have

$$
\begin{aligned}
\left(e_{n} \mid T_{3}\right)(x, y)= & 3 e_{n}(x, y)+e_{n}(3 x, 3 y) \\
& +e_{n}(y, x)+e_{n}(x, y-x)+e_{n}(y-x, y) \\
& +e_{n}(y, x)+e_{n}(x, x+y)+e_{n}(x+y, y)
\end{aligned}
$$

Using the third Manin relation, we see that the bottom two rows of the right hand side vanish. Hence

$$
\left(e_{n} \mid T_{3}\right)(\mathbf{x})=(3+\chi(3)) \cdot e_{n}(\mathbf{x})
$$

and the lemma is proved.

We now return to the proof of the theorem. Let $q=2$ or $q=3$. It follows from the lemma that the Manin symbol $e:=e_{n} \mid\left(T_{q}-(q+\chi(q))\right)$ vanishes on all $\mathbf{x}=(x, y) \in X_{n}$ with $x y \neq 0$. Thus from Proposition 3.8 we see that $e \in$ $\operatorname{Manin}_{\Gamma_{0}\left(p^{n}\right)}^{\infty}\left(\widetilde{K}_{2}\left(R_{n}\right)\right)$ and the theorem is proved.

\section{The Parabolic COHOMOlOGy Class $\varphi_{n}$}

For each positive integer $n$ we let

$$
B_{n}:=\operatorname{Bound}_{\Gamma_{0}\left(p^{n}\right)}\left(\widetilde{K}_{2}\left(R_{n}\right)\right) \text { and } S_{n}:=\operatorname{Symb}_{\Gamma_{0}\left(p^{n}\right)}\left(\widetilde{K}_{2}\left(R_{n}\right)\right)
$$

and consider the exact sequence

$$
0 \longrightarrow B_{n} \longrightarrow S_{n} \stackrel{\pi}{\longrightarrow} H_{\text {par }}^{1}\left(\Gamma_{0}\left(p^{n}\right), \widetilde{K}_{2}\left(R_{n}\right)\right) \longrightarrow 0 .
$$

Let $\phi_{n} \in S_{n}$ be the modular symbol associated to the Manin symbol $e_{n}$ defined in the previous section. We define

$$
\varphi_{n}:=\pi_{\phi_{n}} \in H_{\text {par }}^{1}\left(\Gamma_{0}\left(p^{n}\right), \widetilde{K}_{2}\left(R_{n}\right)\right)
$$

to be the image of $\phi_{n}$. Theorem 1.1 of the Introduction follows now immediately from Theorem 5.1 .

We raise a number of key questions.

\section{Questions:}

(1) Is $\varphi_{n}$ an eigenclass for all the Hecke operators?

(2) Under what conditions can we say $\varphi_{n} \neq 0$ ?

(3) What relations exist among the $\varphi_{n}$ as $n$ varies?

(4) Assuming $\varphi_{n} \neq 0$, is there a Hecke eigensymbol $\psi_{n} \in S_{n}$ lifting $\varphi_{n}$ ?

In fact, in future work, we will draw a connection between the modular symbols $\phi_{n}$ and the Eisenstein distribution [St89] and use this connection to show that $\varphi_{n}$ is an eigenclass satisfying

$$
\varphi_{n} \mid T_{q}=(q+\chi(q)) \cdot \varphi_{n}
$$

for all primes $q$, where we understand that $\chi(q)=0$ when $q=p$. Thus (1) has an affirmative answer.

The answers to questions (2) to (4) are closely tied to some very beautiful recent results of Romyar Sharifi [Sh3-05], which in turn are motivated by work of Ohta (see [Oh03]). Sharifi's ideas suggest that (2) is closely connected to the structure 
of the class group of $K_{n}:=\mathbb{Q}\left(\zeta_{n}\right)$. The answer to (3) should be given (for $m \leq n$ ) in terms of the transfer map $\widetilde{K}_{2}\left(R_{n}\right) \longrightarrow \widetilde{K}_{2}\left(R_{m}\right)$ on $K$-theory composed with corestriction of the cohomology of $\Gamma_{0}\left(p^{n}\right)$ to $\Gamma_{0}\left(p^{m}\right)$. Finally, we expect the answer to (4) to be negative, which corresponds to an expectation that there should be lots of fusion between the boundary cohomology and the parabolic cohomology.

\section{Special values of $L$-Functions}

In this section, following AS86, we define the universal $L$-value of a modular symbol and describe a few of its properties. In particular we will see that the Manin symbols are universal $L$-values.

Let $R$ be a ring and $M$ be an $R$-module endowed with a right action of $S L_{2}(\mathbb{Z})$.

Definition 7.1 (Universal $L$-values). Let $\phi \in H_{c}^{1}\left(S L_{2}(\mathbb{Z}), M\right)$ be a modular symbol. We define $\Lambda(\phi) \in M$ by

$$
\Lambda(\phi):=\phi((\infty)-(0))
$$

and call $\Lambda(\phi)$ the universal $L$-value of $\phi$.

We define $M^{*}$ to be the $R$-dual of $M: M^{*}:=\operatorname{Hom}_{R}(M, R)$ with $S L_{2}(\mathbb{Z})$ acting on the right as $(\lambda \mid \sigma)(m)=\lambda\left(m \mid \sigma^{\prime}\right)$ where $\sigma \mapsto \sigma^{\prime}$ is the adjugate involution $\left(\begin{array}{ll}a & b \\ c & d\end{array}\right) \mapsto$ $\left(\begin{array}{cc}d & -b \\ -c & a\end{array}\right)$. For example, let

$$
W_{r}(R)=\{F \in R[X, Y] \mid F \text { is homogeneous of degree } r\}
$$

with $M_{2}^{+}(\mathbb{Z})$ acting by the formula $(F \mid \sigma)(X, Y)=F\left((X, Y) \sigma^{\prime}\right)$ and let

$$
V_{r}(R):=W_{r}(R)^{*}
$$

As in AS86] we make the following definition.

Definition 7.2. Let $\phi \in H_{c}^{1}\left(S L_{2}(\mathbb{Z}), V_{r}(R)\right)$. We define the special $L$-values $L(\phi, i+1) \in R$ for $i=0,1, \ldots, r$ by

$$
L(\phi, i+1):=\left\langle\Lambda(\phi),(-1)^{i} X^{r-i} Y^{i}\right\rangle
$$

where $\langle\rangle:, V_{r}(R) \times W_{r}(R) \longrightarrow R$ is the canonical pairing.

If $r$ ! is invertible in $R$, we have from Lemma 3.2 of [AS86] a unique $M_{2}^{+}(\mathbb{Z})$ equivariant perfect pairing

$$
\langle,\rangle: W_{r}(R) \times W_{r}(R) \longrightarrow R
$$

with respect to which

$$
\left\langle\left(\begin{array}{c}
r \\
i
\end{array}\right) X^{i} Y^{r-i},(-1)^{j} X^{r-j} Y^{j}\right\rangle= \begin{cases}1 & \text { if } i=j \\
0 & \text { otherwise }\end{cases}
$$

Thus, when $r$ ! is invertible in $R$ we have $V_{r}(R) \cong W_{r}(R)$ and we may regard $\Lambda(\phi)$ as an element of $W_{r}(R)$. With the above identifications we then have

$$
\Lambda(\phi)=\sum_{i=0}^{r}\left(\begin{array}{c}
r \\
i
\end{array}\right) L(\phi, i+1) X^{i} Y^{r-i}
$$

consistent with the conventions of AS86. Note however that Definition 7.2 is meaningful for any commutative ring $R$ - we do not need to assume $r$ ! is invertible in $R$. 
Now consider the general case. Let $\Gamma$ be any congruence subgroup of $S L_{2}(\mathbb{Z})$, let $M$ be a $\Gamma$-module, and let $\varphi \in \operatorname{Symb}_{\Gamma}(M)$ be an $M$-valued modular symbol over $\Gamma$. To define the universal $L$-value of $\varphi$ we first induce to $S L_{2}(\mathbb{Z})$ using Shapiro's Lemma and then take the universal $L$-value of the induced modular symbol.

More precisely, we define the induced module of $M$ to be the module

$$
I(M):=\left\{\begin{array}{l|l}
f: S L_{2}(\mathbb{Z}) \rightarrow M & \left.f(\gamma x)=f(x) \mid \gamma^{-1}, \forall \gamma \in \Gamma, x \in S L_{2}(\mathbb{Z})\right\}
\end{array}\right.
$$

with $S L_{2}(\mathbb{Z})$ acting by $(f \mid g)(x)=f\left(x g^{-1}\right)$. The Shapiro isomorphism gives us a canonical isomorphism

$$
H_{c}^{1}(\Gamma, M) \stackrel{I}{\longrightarrow} H_{c}^{1}\left(S L_{2}(\mathbb{Z}), I(M)\right),
$$

which is given explicitly on modular symbols by $I: \varphi \longmapsto I(\varphi)$, where $I(\varphi)$ : $\Delta_{0} \longrightarrow I(M)$ is given by

$$
I(\varphi)(D)(x)=\varphi(x D)
$$

for $D \in \Delta_{0}$ and $x \in S L_{2}(\mathbb{Z})$.

Definition 7.3. Let $\varphi \in H_{c}^{1}(\Gamma, M)$. Then the universal $L$-value of $\varphi$ is defined to be

$$
\Lambda(\varphi):=I(\varphi)((\infty)-(0))
$$

In other words we set $\Lambda(\varphi)=\Lambda(I(\varphi))$.

In the special case where $M$ is a $\Gamma_{0}\left(p^{n}\right)$-module on which $\Gamma_{0}\left(p^{n}\right)$ acts via a nebentype character $\chi$ we may identify $I(M)$ with the module of functions $f$ : $X_{n} \longrightarrow M$ satisfying $f(d \mathbf{x})=\chi(d) \cdot f(\mathbf{x})$, which we will denote by $I_{\chi}(M)$. Thus we have a natural inclusion

$$
\operatorname{Manin}_{\Gamma_{0}\left(p^{n}\right)}(M) \hookrightarrow I(M) .
$$

In fact, we have the following simple proposition, whose proof we leave to the reader.

Proposition 7.4. Let $M$ be an R-module on which $\Sigma_{0}\left(p^{n}\right)$ acts via a nebentype character $\chi$ and let $\phi \in H_{c}^{1}\left(\Gamma_{0}\left(p^{n}\right), M\right)$ be a modular symbol. Then with the above identifications, we have

$$
\Lambda(\phi)=\mathbf{e}_{\phi} .
$$

Finally, we turn to the problem of defining special $L$-values of parabolic cohomology classes. For this we need to understand the module $V_{r}(R)^{\Gamma_{\infty}}$. For the rest of this section $R$ will be a ring of characteristic $p$ and $r \geq 0$ will an even integer. Let $\left\{\lambda_{i}\right\}_{i=0}^{r}$ in $V_{r}(R)$ be the dual basis to $\left\{(-1)^{i} X^{r-i} Y^{i}\right\}_{i=0}^{r}$ in $W_{r}(R)$. We leave the simple proof of the following lemma to the reader.

Lemma 7.5. Let $r$ be an even integer. If $r<p$, then $\lambda_{r}$ spans $V_{r}(R)^{\Gamma_{\infty}}$. If $p \leq r<2 p$, then $\lambda_{r}$ and $\lambda_{p-1}$ span $V_{r}(R)^{\Gamma_{\infty}}$.

Proposition 7.6. Let $\Gamma=S L_{2}(\mathbb{Z})$, let $i, r$ be positive integers with $r$ even and $0 \leq i \leq r$. Let $\varphi \in \operatorname{Bound}_{\Gamma}\left(V_{r}(R)\right)$ be a boundary symbol. Then

(a) If $r<2 p$, then $L(\varphi, i+1)=0$ for $i \not \equiv 0, r(\bmod p-1)$.

(b) For arbitrary $r$, if $\varphi \mid T_{p}=\varphi$, then $L(\varphi, i+1)=0$ for $i \neq 0, r$. 
Proof. Fix $i, r$ as in the statement of the proposition and let $\varphi \in \operatorname{Bound}_{\Gamma}\left(V_{r}(R)\right)$. Since $S L_{2}(\mathbb{Z})$ has only one cusp, we have $\varphi=\varphi_{\infty, \lambda}$ for some $\lambda \in V_{r}(R)^{\Gamma_{\infty}}$. By Lemma 7.5 there are constants $a, b \in R$ such that $\lambda=a \lambda_{r}$, if $r<p$, and $\lambda=$ $a \lambda_{r}+b \lambda_{p-1}$ if $p<r<2 p$. But then

$$
\Lambda(\varphi)=\lambda-\lambda \mid\left(\begin{array}{cc}
0 & -1 \\
1 & 0
\end{array}\right)= \begin{cases}a\left(\lambda_{r}-\lambda_{0}\right) & \text { if } r<p, \\
a\left(\lambda_{r}-\lambda_{0}\right)+b\left(\lambda_{p-1}-\lambda_{r-p+1}\right) & \text { if } p<r<2 p .\end{cases}
$$

For $0 \leq i \leq r$ we have that $L(\varphi, i+1)$ is the coefficient of $\lambda_{i}$, and by inspection we have, for $r<2 p$,

$$
L(\varphi, i+1)=0
$$

for $i \not \equiv 0, r(\bmod p-1)$. This proves $(\mathrm{a})$.

To prove (b) we write $\lambda=\sum_{i=0}^{r} a_{i} \lambda_{i}$ with coefficients in $R$ and let $m$ be the smallest index for which $a_{m} \neq 0$.

We remark that

$$
\lambda_{i} \mid T_{p}=\lambda_{i}\left(p^{i+1}+p^{r-i}\right)+\sum_{j=i+1}^{r}(-1)^{j-i} \lambda_{j}\left(\begin{array}{c}
r-i \\
r-j
\end{array}\right) \sum_{k=0}^{p-1} k^{j-i} .
$$

Since $\varphi \mid T_{p}=\varphi$ we have

$$
\lambda=\left(\varphi \mid T_{p}\right)(\infty)=\left(\varphi \mid\left(\begin{array}{ll}
p & 0 \\
0 & 1
\end{array}\right)\right)(\infty)+\sum_{k=0}^{p-1}\left(\varphi \mid\left(\begin{array}{ll}
1 & k \\
0 & p
\end{array}\right)\right)(\infty)=\lambda \mid\left(\left(\begin{array}{ll}
p & 0 \\
0 & 1
\end{array}\right)+\sum_{k=0}^{p-1}\left(\begin{array}{ll}
1 & k \\
0 & p
\end{array}\right)\right) .
$$

Comparing the coefficients of $\lambda_{m}$ on both sides of this equation we obtain $p^{m+1}+$ $p^{r-m}=1$ in the ring $R$. But since $p=0$ in $R$ this can only happen if $r=m$.

Definition 7.7. For $\psi \in H_{\text {par }}^{1}\left(\Gamma, V_{r}(R)\right)$, with $r<2 p$, we choose $\widetilde{\psi} \in$ $\operatorname{Symb}_{\Gamma}\left(V_{r}(R)\right)$ to be an arbitrary lift of $\psi$ and define

$$
L(\psi, i+1)=L(\widetilde{\psi}, i+1) \text { for } 0 \leq i \leq r \text { with } i \not \equiv 0, r(\bmod p-1) .
$$

We call these the special $L$-values of $\psi$.

By (a) of Proposition 7.6, these special $L$-values are well defined, independent of the choice of $\widetilde{\psi}$. In fact, by (b) of the proposition, if $\widetilde{\psi} \mid T_{p}=\widetilde{\psi}$, then all of the $L$-values $L(\psi, i+1)$ are well defined in the range $0<i<r$.

\section{Proof of Theorem 1.2}

In this section we prove Theorem 1.2 of the introduction. We take $n=1$ and suppress the subscript $n$ from the notation. Thus $\zeta=\zeta_{1}$ is a primitive $p^{\text {th }}$ root of unity, $R=R_{1}=\mathbb{Z}\left[\zeta, \frac{1}{p}\right], G=G_{1}$ is the Galois group of $\mathbb{Q}(\zeta) / \mathbb{Q}, \phi=\phi_{1}, \varphi=\varphi_{1}$, $e=e_{1}$, and $X=X_{1}=\left(\mathbb{F}_{p}^{2}\right)^{\prime}$, and for arbitrary $d$ we let $I_{d}=I_{\omega^{d}}\left(\mathbb{F}_{p}\right), V_{d}=V_{d}\left(\mathbb{F}_{p}\right)$, and $W_{d}=W_{d}\left(\mathbb{F}_{p}\right)$.

We also let $k \geq 2$ be an even integer and set $g=2-k$. The semigroup $M_{2}^{+}(\mathbb{Z})$ acts on $W_{k-2}$ and $I_{d}$ (for any $d$ ) by

$$
\begin{aligned}
(F \mid \sigma)(X, Y) & :=F\left((X, Y) \sigma^{\prime}\right), \\
(f \mid \sigma)(\mathbf{x}) & :=f\left(\mathbf{x} \sigma^{\prime}\right)
\end{aligned}
$$

for $F \in W_{k-2}, f \in I_{d}$, and $\sigma \in M_{2}^{+}(\mathbb{Z})$, where in the latter case we take $f\left(\mathbf{x} \sigma^{\prime}\right)=0$ in case $\mathbf{x} \sigma^{\prime}=0$. 
We will use the notation $M(g):=M \otimes \operatorname{det}^{g}$ for any $M_{2}^{+}(\mathbb{Z})$-module $M$.

The pairing

$$
\begin{aligned}
I_{k-2} \times I_{g} & \longrightarrow \mathbb{F}_{p} \\
\left(f_{1}, f_{2}\right) & \longmapsto \sum_{\mathbf{x} \in X} f_{1}(\mathbf{x}) f_{2}(\mathbf{x})
\end{aligned}
$$

can be seen to induce the isomorphism

$$
I_{g} \cong I_{k-2}^{*}(g) .
$$

There is a natural map of $M_{2}^{+}(\mathbb{Z})$-modules: $W_{k-2} \longrightarrow I_{k-2}$ defined by sending a polynomial to the function it represents. In [AS86 it is shown that this map is injective if $k-2<p$ and is surjective otherwise. By duality we obtain a natural map

$$
I_{g} \stackrel{\beta}{\longrightarrow} V_{k-2}(g)
$$

which is surjective if $k-2<p$ and injective otherwise.

One can easily check that the map $\beta$ is given explicitly by

$$
\beta(f)=\sum_{i=0}^{k-2} \lambda_{i} \sum_{(x, y) \in X}(-1)^{i} x^{k-2-i} y^{i} f(x, y),
$$

where the $\lambda_{i}$ 's were defined in the previous section.

For more details, see Lemma 3.2 of AS86.

We now turn to the proof of Theorem 1.2. So, suppose $2 \leq k \leq 2 p$ and that Hypothesis $H_{k}$ from the introduction is satisfied. Thus we have a $G$-equivariant map

$$
\rho: K_{2}(R) \rightarrow \mathbb{F}_{p}\left(\omega^{g}\right)
$$

and an odd integer $i \neq p$ with $1<i<k-1$ such that $\rho\left(\xi_{i}\right) \neq 0$. Here $\xi_{i}:=\left\{\eta_{k-i}, \eta_{i}\right\}$ as in the introduction.

We let $\Gamma:=S L_{2}(\mathbb{Z})$ and define the map

$$
\lambda: H_{c}^{1}\left(\Gamma_{0}, \widetilde{K}_{2}(R)\right) \longrightarrow H_{c}^{1}\left(\Gamma, V_{k-2}\right)
$$

to be the composition

$$
\lambda: H_{c}^{1}\left(\Gamma_{0}, \widetilde{K}_{2}(R)\right) \stackrel{S h_{\rho}}{\longrightarrow} H_{c}^{1}\left(\Gamma, I_{g}\right) \stackrel{\nu}{\longrightarrow} H_{c}^{1}\left(\Gamma, V_{k-2}\right),
$$

where $S h_{\rho}$ is the map induced by $\rho$ and the Shapiro isomorphism, and $\nu$ is the composition of $\beta$ and the "twist map"

$$
H_{c}^{1}\left(\Gamma, V_{k-2}(g)\right) \stackrel{\tau}{\longrightarrow} H_{c}^{1}\left(\Gamma, V_{k-2}\right),
$$

which is defined as the identity map on the underlying cohomology groups. Note, however, that $\tau$ does not commute with the action of the Hecke operators $T_{m}$. Indeed, we have $\tau\left(\varphi \mid T_{m}\right)=m^{g} \cdot \tau(\varphi) \mid T_{m}$ for every $\varphi \in H_{c}^{1}\left(\Gamma, V_{k-2}(g)\right)$.

Now let $\phi$ be the modular symbol defined in Section 5 and set

$$
\widetilde{\psi}:=\lambda(\phi) \in H_{c}^{1}\left(\Gamma, V_{k-2}\right) \quad \text { and } \quad \psi:=\text { the image of } \widetilde{\psi} \text { in } H_{p a r}^{1}\left(\Gamma, V_{k-2}\right) .
$$

It is proved in AS86 that $S h_{\rho}$ is Hecke equivariant, so from the last paragraph we have

$$
m^{g} \cdot \widetilde{\psi} \mid T_{m}=\lambda\left(\phi \mid T_{m}\right)
$$


for every $m \in \mathbb{N}$. In particular, for $q=2,3$ we have

$$
\begin{aligned}
\psi \mid T_{q} & =q^{k-2} \lambda((q+\chi(q)) \cdot \phi)=q^{k-2}\left(q+q^{g}\right) \cdot \psi \\
& =\left(1+q^{k-1}\right) \psi,
\end{aligned}
$$

proving (1) of Theorem 1.2.

To compute the special $L$-values $L(\psi, i+1)$ for $2 \leq i<k-2$ we use Proposition 7.4 to conclude that $\Lambda\left(S h_{\rho}(\phi)\right)=\rho \circ e$, where $e$ was defined in Theorem 5.1. Thus $\Lambda\left(S h_{\rho}(\phi)\right)$ is the function

$$
\begin{aligned}
\Lambda\left(S h_{\rho}(\phi)\right): & X \\
(x, y) & \longmapsto \mathbb{F}_{p} \\
& \longmapsto\left(\left\{1-\zeta^{x}, 1-\zeta^{y}\right\}\right) \text { if } x y \neq 0 .
\end{aligned}
$$

Then $\Lambda(\widetilde{\psi})=\beta\left(\Lambda\left(S h_{\rho}(\phi)\right)\right)$. Thus, from the explicit description of the map $\beta$, we have

$$
\Lambda(\widetilde{\psi})=\sum_{i=0}^{k-2} \lambda_{i} \sum_{(x, y) \in X}(-1)^{i} x^{k-2-i} y^{i} \rho(e(x, y)),
$$

which by the definition of $L$-values (Definition 7.2) means

$$
\begin{aligned}
(-1)^{i} L(\widetilde{\psi}, i+1) & =\sum_{(x, y) \in\left(\mathbb{F}_{p}^{\times}\right)^{2}} y^{i} x^{k-2-i} \rho\left(\left\{1-\zeta^{x}, 1-\zeta^{y}\right\}\right) \\
& =\rho\left(\left\{\prod_{x \in \mathbb{F}_{p}^{\times}}\left(1-\zeta^{x}\right)^{x^{k-2-i}}, \prod_{y \in \mathbb{F}_{p}^{\times}}\left(1-\zeta^{y}\right)^{y^{i}}\right\}\right) \\
& =\rho\left(\left\{\prod_{\sigma \in G}(1-\zeta)^{\omega^{k-2-i}(\sigma) \sigma}, \prod_{\sigma \in G}(1-\zeta)^{\omega^{i}(\sigma) \sigma}\right\}\right) .
\end{aligned}
$$

Finally, we recall that $\xi_{j} \in K_{2}(R)$ was defined in the Introduction as $\xi_{j}:=$ $\left\{\eta_{k-j}, \eta_{j}\right\}$ where $\eta_{j} \in E_{j}$ is the projection of $(1-\zeta)$ to $E_{j}:=E^{(1-j)}$. Recalling that the idempotent projecting to $E^{(1-j)}$ is $\frac{1}{p-1} \sum_{\sigma \in G} \omega^{j-1}(\sigma) \sigma$, we conclude that

$$
\eta_{j}^{-1}=\prod_{\sigma \in G}(1-\zeta)^{\omega^{j-1}(\sigma) \sigma} .
$$

Thus

$$
(-1)^{i} L(\widetilde{\psi}, i+1)=\rho\left(\left\{\eta_{k-1-i}^{-1}, \eta_{i+1}^{-1}\right\}\right)=\rho\left(\xi_{i+1}\right)
$$

for $i=0, \ldots, k-2$. The $L$-values of $\psi$ are the same as those for $\widetilde{\psi}$, but with the values at $i+1$ with $i \equiv 0, k-2(\bmod p-1)$ excluded. Moreover, $\xi_{j}=0$ for even $j$, so for $2 \leq i<k-2, i \neq p$, we have proved

$$
L(\psi, i)= \begin{cases}\rho\left(\xi_{i}\right) & \text { if } i \text { is odd } \\ 0 & \text { otherwise. }\end{cases}
$$

This completes the proof of Theorem 1.2.

\section{Sharifi's Conjecture}

Let $f$ be a weight $k \geq 2$ cusp form whose Fourier expansion is given by $f(z)=$ $\sum_{n \geq 1} a_{n} e^{2 \pi i n z}$. Let $L(f, s)$ be the complex $L$-function of $f$. Then, $f$ gives rise to a class $\phi_{f} \in H_{c}^{1}\left(S L_{2}(\mathbb{Z}), V_{k-2}(\mathbb{C})\right)$ given by

$$
\phi_{f}((x)-(y))=\int_{y}^{x} f(z)(z X+Y)^{k-2} d z .
$$


It is well known that $L\left(\phi_{f}, \alpha\right)=\frac{(-1)^{\alpha-1}(\alpha-1) !}{(2 \pi i)^{\alpha}} L(f, \alpha)$ for all integers $\alpha$ with $1 \leq$ $\alpha \leq k-1$.

We will denote by $\psi_{f}$ the parabolic cohomology class associated to $\phi_{f}$ in $H_{\text {par }}^{1}\left(S L_{2}(\mathbb{Z}), V_{k-2}(\mathbb{C})\right)$.

Now let $p$ be an irregular prime and choose $k, 2 \leq k<p$, such that $p \mid \frac{B_{k}}{k}$. Let $f$ be a normalized weight $k$ newform of level 1 and let $\mathcal{O}_{f}$ be the ring of integers of the number field $K_{f}$ generated by the Fourier coefficients of $f$. Let $\wp$ be a place above $p$ and assume $f \equiv G_{k}(\bmod \wp)$, where $G_{k}$ is the Eisenstein series of level 1 and weight $k$ : $G_{k}=-\frac{B_{k}}{2 k}+\sum_{n=1}^{\infty} \sigma_{k-1}(n) q^{n}$ with $\sigma_{k-1}(n)=\sum_{d \mid n, d>0} d^{k-1}$.

The following is a Conjecture of Romyar Sharifi, which was mentioned in this form in Sh1-04].

Conjecture (Sharifi). Under the above hypotheses, there exists $\Omega \in \mathbb{C}^{\times}$such that $\frac{L\left(\psi_{f}, i\right)}{\Omega} \in \mathcal{O}_{f}$ for all odd $i$ in the range $3 \leq i \leq k-3$, at least one of these numbers is non-zero modulo $\wp$, and there exists $\rho$ such that hypothesis $H_{k}$ holds for the irregular pair $(p, k)$ and

$$
\frac{L\left(\psi_{f}, i\right)}{\Omega} \equiv \rho\left(\xi_{i}\right)(\bmod \wp)
$$

for all odd $i$ in the range $3 \leq i \leq k-3$.

We remark that the computations of McCallum and Sharifi (see MS03, Theorem 5.1 , [MS]) imply that the space $H_{k, e i s,\{2,3\}}^{+}$defined in the Introduction is at most one-dimensional for all irregular pairs $(p, k)$ with $p<10,000$. In fact, McCallum and Sharifi only compute the equivalent of the eigenvalue of $T_{2}$, so in this range, we even have the stronger statement

$$
\operatorname{dim}_{\mathbb{F}_{p}}\left(H_{k, e i s,\{2\}}^{+}\right) \leq 1 .
$$

In [Sh4-06, the above Conjecture is claimed to be true for any $k$. However, our methods do not give any information on the $L$-values corresponding to the points in which we encounter interferences with the boundary symbols (see the proof of Theorem 1.2).

\section{REFERENCES}

[AS86] Ash, A., Stevens, G.: Modular forms in characteristic $\ell$ and special values of their $L$ functions, Duke Mathematical Journal 53, No. 3, 1986. MR860675 (88h:11036)

[GS91] Greenberg, R., Stevens, G.: On the conjecture of Mazur, Tate, and Teitelbaum, in $p$-adic Monodromy and the Birch and Swinnerton-Dyer Conjecture (Contemporary Mathematics 165), B. Mazur and G. Stevens, eds., 1991. MR1279598 (94m:11006)

[Ma72] Manin, J.: Parabolic Points and Zeta Functions of Modular Curves, Math. USSR Izvestija 36, No. 1, 1972, 19-66. MR0314846 (47:3396)

[Ma73] Manin, J.: Periods of parabolic forms and p-adic Hecke series, Math. USSR Sbornik 21, No. 3, 1973.

[MS03] McCallum, W., Sharifi, R.: A Cup Product in Galois Cohomology, Duke Mathematical Journal 120, No. 2, 2003. MR2019977 (2004j:11136)

[MS] McCallum, W., Sharifi, R.: Magma routines for computing the table of pairings for $p<$ 1000, http://abel.math.harvard.edu/ sharifi/computations.html, http://math.arizona. $\mathrm{edu} / \sim \mathrm{wmc} 284$.

[Mer94] Merel, L.: Universal Fourier expansions of modular forms, On Artin's conjecture for odd 2-dimensional representations, Springer, Berlin, 1994, pp. 59-94. MR1322319 (96h:11032)

[Mi71] Milnor, J.: Introduction to Algebraic K-theory, Annals of Mathematics Studies 72, Princeton University Press, 1971. MR 0349811 (50:2304) 
[Oh03] Ohta, M.: Congruence modules related to Eisenstein series, Ann. Scient. École Norm. Sup., $4^{\mathrm{e}}$ série 36 (2003), 225-269. MR.1980312 (2004d:11045)

[Sh1-04] Sharifi, R.: The various faces of a pairing on $p$-units, slides from a talk at International Univ. Bremen on 5/10/04, http://www.math.mcmaster.ca/ sharifi/bremen.pdf.

[Sh2-04] Sharifi, R.: Computations on Milnor's $K_{2}$ of Integer Rings, slides from a talk at Max Planck Institute of Mathematics on 5/17/04, http://www.math.mcmaster. ca/ sharifi/dagslides.pdf.

[Sh3-05] Sharifi, R.: Iwasawa Theory and the Eisenstein Ideal, Duke Math. J. 137 (2007), 63-101. MR2309144

[Sh4-06] Sharifi, R.: Cup Products and $L$-values of Cusp Forms, preprint.

[St89] Stevens, G.: The Eisenstein measure and real quadratic fields, in The Proceedings of the International Number Theory Conference (Université Laval, 1987), J.-M. De Koninck and C. Levesque, eds., de Gruyter, 1989. MR1024612 (90m:11077)

[W97] Washington, L.C.: Introduction to Cyclotomic Fields, Graduate Texts in Mathematics 83, Springer, New York, 1997. MR1421575 (97h:11130)

Department of Mathematics, Boston University, 111 Cummington Street, Boston, MASSACHUSETTS 02215

E-mail address: celiab@math.bu.edu 